\title{
NUTRICIÓN
}

\section{Hábitos alimentarios y estilos de vida en estudiantes del 3er ciclo de la EEB del Colegio Iberoamericano}

\author{
Andrea Villalba ${ }^{1}$ Humberto García ${ }^{1}$
}

\begin{abstract}
Resumen
Introducción: El aumento de la producción de alimentos procesados, la rápida urbanización y el cambio en los estilos de vida han dado lugar a un cambio en los hábitos alimentarios. Actualmente, las personas consumen más alimentos hipercalóricos, grasas, azúcares libres y sal/sodio; disminuyendo la ingesta de frutas, verduras y fibra dietética, como por ejemplo cereales integrales. Los programas y políticas escolares son clave para lograr los derechos humanos de los niños a la alimentación, la educación y la salud. A través de intervenciones complementarias, como comidas escolares saludables y educación sobre alimentación y nutrición, los alumnos pueden mejorar sus dietas, desarrollar prácticas alimentarias más sanas y extenderlas a sus familias y comunidades.

Objetivo: Determinar hábitos alimentarios y estilos de vida en Estudiantes del 3er ciclo de la EEB del Colegio Iberoamericano, Junio 2019.

Material y Método: El trabajo de investigación presentó un diseño no experimental, descriptivo de corte transversal, enfoque cuantitativo. La población utilizada estudiantes de ambos sexos del tercer ciclo de la educación escolar básica, la muestra estuvo conformada por 18 alumnos del tercer ciclo de la EEB. El Criterios de inclusión fue de aquellos estudiantes que acepten participar del estudio. Se realizó en base a la técnica de la encuesta, basado en un instrumento, siendo un cuestionario previamente elaborado que contemplan todas las variables en estudio. El análisis se realizó a través del programa Excel, con lo cual se cargaron los datos por medio de estadística descriptiva y se utilizaron tablas y gráficos.
\end{abstract}

Resultados: El 67\% de los adolescentes (12 estudiantes) cree tener una buena alimentación en comparación al 33\% (6 estudiantes). El 28\% de los encuetados ( 5 alumnos) se siente gordo, se ven flacos el $22 \%$ (4 alumnos) y se ven normales el 50\% (9 alumnos). El 89\% de los encuestados lleva una dieta libre sin restricciones y solo el $39 \%$ realiza las 4 comidas principales, y tienen

1. Universidad Iberoamericana. Facultad de Ciencias de la Salud, Paraguay.

E-mail: nutricionunibe17@gmail.com

DOI: $10.26885 /$ rcei.foro.2019.233

Trabajo publicado en acceso abierto bajo Licencia Creative Commons. 
suplementación vitamínica el 17\%. El 56\% de los encuetados consume lácteos con frecuencia, el 39\% consume carne vacuna 3 veces por semana, el 33\% consume fruta durante la semana y consumen verduras con frecuencia el $39 \%$. Sólo el 50\% realiza actividad física diaria y se hidratan con 5 a 7 vasos de agua por día el 55\% de los estudiantes.

Conclusión: Dando como resultado favorable el porcentaje de adolescentes que no tienen ninguna restricción dietaría, y resultados preocupantes en porcentajes bajos de consumo de lácteos, frutas, verduras, agua, sin omitir la escasa actividad física que desarrollan. Algunos se suplementan con vitaminas, mientras que otros alumnos no consume ningún tipo de suplementos. La mayoría están interesados en aprender sobre nutrición. Aunque esta etapa del ser humano es de crecimiento, constituye también riesgos considerables, durante la cual el contexto social puede tener una influencia determinante Se confirma que los datos proveídos por la Dirección de Vigilancia de Enfermedades No Transmisibles, el 57\% de la población mayor de 20 años tiene sobrepeso y obesidad, afectando más frecuentemente a las mujeres. Esto tiene una directa relación con la mala alimentación y la falta de actividad física en etapa adolescente.

Palabras clave: hábitos, estilos de vida, estudiantes.

\section{REFERENCIAS}

López, L., Suárez, M. (2017). Fundamentos de la Nutrición Normal. Buenos Aires: Editorial El Ateneo.

Organización Mundial de la Salud. (2019). Alimentación Sana. https://www. who.int/es/news-room/fact-sheets/detail/healthy-diet

Organización Mundial de la Salud. (sf). Desarrollo en la adolescencia. https:// www.who.int/maternal_child_adolescent/topics/adolescence/dev/ es/

Organización para las Naciones Unidas para la Alimentación y Cultura. (2018). Alimentación y Nutrición Escolar. http://www.fao.org/school-food/ es/ 